\title{
Suez 1869: un cosmopolitisme eurocentré?
}

\section{Le témoignage de quelques voyageurs français}

Résumé: Cet article propose l'hypothèse selon laquelle l'ouverture du canal de Suez, célébré par nombre de témoins, en 1869, comme le «mariage de l'Orient et de l'Occident », selon la formule de l'historien de l'art Charles Blanc, fut en réalité la mise en scène d'un cosmopolitisme eurocentré. Le rêve du creusement de l'isthme de Suez remonte à l'antiquité pharaonique. Mais c'est Bonaparte qui, dans l'histoire moderne, lui redonna un nouvel élan, relayé par le projet « civilisateur » des saint-simoniens, dont le jeune Ferdinand de Lesseps fut luimême proche. On s'intéressera au discours d'une utopie réconciliatrice chez un certain nombre de voyageurs français invités par le khédive Ismaill (Blanc, Taglioni, la Bédollière, Pharaon...) - discours reposant sur une profonde asymétrie, puisqu'il conduisit, dans les faits, à la ruine de l'État égyptien, donc à son affaiblissement, qui en fit une proie facile pour l'occupant anglais, en 1882. On rappelle également les « absents » de l'histoire - les fellahs (les paysans égyptiens qui, les premiers, creusèrent le canal dans des conditions très difficiles), bien peu présents dans les récits des voyageurs français, mais aussi les Arabes (au mieux réduits textuellement à une scénographie pittoresque, au pire symptômes des préjugés ethnocentriques caractéristiques du «discours orientaliste » dénoncé par E. Said), enfin les invités orientaux eux-mêmes aux fêtes de Suez, lesquels se comptaient par centaines, si ce n'est par milliers, mais dont il reste à retrouver les témoignages, au-delà de leurs représentations plus ou moins instrumentalisées, ainsi la figure d'Abd el-Kader, dans un journal comme L'Isthme de Suez. Journal de l'union des deux mers. On termine en évoquant un contre-discours en creux, celui de Théophile Gautier, qui ne mentionne pas une seule fois (silence assourdissant!) le nom de Lesseps dans les feuilletons de son séjour en Égypte, en 1869.

Mots-clés: canal de Suez; Bonaparte; saint-simoniens; voyageurs; orientalisme; utopie; cosmopolitisme

En 1869, le canal de Suez était ouvert, à la suite d'une dizaine d'années d'un chantier qui constitua, comme le dit le catalogue d'une exposition de l'Institut du

Sarga Moussa, CNRS/Université Sorbonne Nouvelle-Paris 3

2 Open Access. ( 2021 Sarga Moussa (c) BY-NC-ND This work is licensed under a Creative Commons Attribution-NonCommercial-No-Derivatives 4.0 License.

https://doi.org/10.1515/9783110691504-005 
Monde Arabe consacrée à ce sujet, une véritable « épopée » (Gauthier et Mollard 2018). Cette histoire a été longtemps racontée comme le triomphe d'un monde enfin réconcilié (l'annonce, déjà, d'une illusoire « fin de l'Histoire »?), sous l'égide d'un commerce mondial à travers lequel les peuples s'enrichiraient mutuellement, voire fantasmée comme le «mariage » de l'Orient et de l'Occident - une métaphore qu'on trouve, par exemple, dans le récit de voyage de l'historien de l'art Charles Blanc, qui figure parmi les nombreux invités du khédive Ismaïl pour les fêtes d'inauguration du canal de Suez ${ }^{1}$ - j'y reviendrai. Certes, plus personne n'est assez naïf, aujourd'hui, pour penser l'histoire des relations franco-égyptienne, surtout à la fin du $\mathrm{XIX}^{\mathrm{e}}$ siècle, c'est-à-dire à l'époque des impérialismes coloniaux (Said 1980), comme une idylle, mais on trouve parfois, dans les ouvrages les plus récents, les échos tardifs de cet imaginaire idéalisant du mélange culturel qu'aurait généré Suez, par exemple lorsqu'il est question d' " une forme particulière de cosmpolitisme [...] dont persiste la nostalgie ", comme l'écrivent les commissaires de l'exposition de l'IMA déjà citée, non sans reconnaître avec honnêteté, dans la même phrase, l'existence " des injustices et des inégalités » associées au même événement ${ }^{2}$. Je voudrais ici, dans la lignée du dossier sur « Les imaginaires du canal de Suez » paru dans Sociétés et Représentations (Moussa et Sabry 2019), m'interroger sur les asymétries et les non-dits associés à l'imaginaire cosmopolitique des voyageurs invités aux fêtes du canal de Suez. On commencera par rappeler le souvenir de l'expédition de Bonaparte, médiatisé par l'expérience saint-simonienne des années 1830 en Égypte. On examinera ensuite le discours d'une utopie réconciliatrice, avant de pointer les absents de l'Histoire et d'évoquer brièvement, en forme de contre-point, une voix discordante, celle de Théophile Gautier, invité aux fêtes de 1869, mais qui ne dit pas un mot de Ferdinand de Lesseps, pourtant célébré dans tous les discours officiels comme le grand architecte du canal de Suez.

\section{De Napoléon à Lesseps}

On sait que l'histoire du canal remonte à l'Antiquité, puisque le pharaon Sésostris III (Moyen Empire, $\mathrm{XIX}^{\mathrm{e}}$ siècle avant J.-C.), avait déjà eu le projet de relier la mer Rouge au Nil et avait même fait creuser pour cela un canal, qui s'ensabla néanmoins avec le temps. En arrivant en Égypte en 1798, Bonaparte reprit cette idée

\footnotetext{
1 Charles Blanc estime à « mille environ » le total des invités, précisant qu'il ne s'agit que des Européens (1876, 341). Ce chiffre est repris par Jean-Marie Carré (1990 II [1956], 307).

2 Gauthier et Mollard, « Un canal quatre fois millénaire » $(2018,17)$; je souligne.
} 
en lui donnant plus d'ampleur encore, comprenant l'importance stratégique que pourrait jouer un canal reliant cette fois-ci la mer Méditerranée à la mer Rouge, ce qui permettrait de contrôler la route des Indes, donc de faire directement concurrence au grand rival anglais. Une commission, présidée par l'ingénieur Jean-Marie Le Père, fut chargée d'étudier la faisabilité de ce projet. Dans un Mémoire publié dans la Description de l'Égypte (1822 [1809]), Le Père concluait qu'un canal pourrait bel et bien être creusé, mais qu'il faudrait y installer des écluses, car, pensait-il à tort, il y avait une différence de niveau entre la Méditerranée et la mer Rouge. C'est cette erreur de calcul qui conduisit Bonaparte à abandonner le projet, dont il était entendu, pour lui, qu'il n'aurait pu être mené à bien que sous l'égide européenne. Comme l'écrivait Le Père: « Les peuples mahométans qui habitent des pays plus voisins à la fois de l'Inde et des bords de la Méditerranée, opprimés par des gouvernements barbares et étrangers à toute idée de perfectionnement et de civilisation, ont langui dans l'indolence, qui en est l'effet ordinaire (Le Père 1822 [1809], 48) ». Le discours d'une Égypte endormie par des siècles d'occupation ottomane mais heureusement réveillée par la secousse post-révolutionnaire de l'expédition de Bonaparte, on le sait, constitue aujourd'hui encore le cadre de pensée d'une historiographie souvent très eurocentriste ${ }^{3}$.

Je pars de l'hypothèse que la « légende de Bonaparte », dont on sait qu'elle marqua un certain nombre d'écrivains, en particulier sous Louis-Philippe (Hugo, Stendhal, Balzac... $)^{4}$, continue de hanter les esprits à l'époque de la construction du canal de Suez. J'en veux pour preuve cette page écrite par Ferdinand de Lesseps et figurant au tome $\mathrm{V}$ de l'ensemble de documents qu'il rassembla sur le tard sous le titre de Lettres, journal et documents pour servir à l'histoire du canal de Suez: « Le 15 [août 1865], la Saint-Napoléon a été fêtée à Ismaiilia par l'arrivée du convoi de Port-Saïd et par l'ouverture de l'écluse qui a offert un spectacle magnifique » (Lesseps 1881, 179). La Saint-Napoléon, décrétée par l'empereur lui-même en 1806 pour fêter le rétablissement de la religion catholique en France par la signature du Concordat (1801), coïncidait en même temps, non par hasard, avec le jour de la naissance de Napoléon. Considérée comme fête nationale sous le Second Empire également, elle ancrait dans la mémoire collective le souvenir du héros et de ses conquêtes, donc une mythologie de la grandeur que la France de Napoléon III cherchait, elle aussi, à entretenir, y compris dans le cadre du percement de l'isthme de Suez, comme en témoigne Lesseps dans la suite de la même page précédemment citée :

3 Voir ici même la contribution de Leyla Dakhli.

4 Descotes (1967). Sur le mythe de Napoléon, voir par ailleurs les nombreux travaux de l'historien Jean Tulard. 
J'avais, dès le matin, télégraphié ce qui suit à l'Empereur:

«Sa Majesté fêtée dans l'Isthme par première cargaison de houille transitant directement de Méditerranée à mer Rouge. - Signé: LESSEPS »

Cette dépêche était partie de Suez pour Paris, le 15, à 8 heures du matin.

Le même jour arrivait à Suez, à 5 heures du soir, la réponse de l’Empereur, ainsi conçue:

« Napoléon,

À M. de Lesseps.

Je vous félicite du succès obtenu et vous remercie de me donner cette bonne nouvelle pour ma fête » (Lesseps 1881, 179-180).

On aura remarqué la signature de l'Empereur, dans ce télégramme: le nom de « Napoléon » permet la superposition avec celui de l’oncle, Lesseps inscrivant du même coup sa propre entreprise sous le signe d'une histoire doublement impériale, mais renvoyant surtout au prestige de Napoléon $\mathrm{I}^{\mathrm{er}}$, lequel avait en quelque sorte anticipé, par son projet de canal de Suez, l'idée d'une rencontre, voire d'une fusion entre l'Orient et l'Occident.

On sait que les saint-simoniens, partis en Égypte sous la houlette d'Émile Barrault, puis du « Père » Enfantin qui les y rejoignit un peu plus tard, en 1833, envisagèrent eux aussi le creusement de l'isthme de Suez ${ }^{5}$. C'est même le premier projet que ce mouvement à la fois utopiste et matérialiste proposa à Mohammad Ali, qui s'y opposa, craignant que son pays ne perde son autonomie. Mais le vice-roi francophile et modernisateur n'était pas opposé à l'idéologie progressiste des saintsimoniens, tout au contraire, et il approuva, dans un second temps, la construction d'un barrage sur le Nil, à l'endroit où le Delta se sépare en deux branches. Barrault, dans Occident et Orient (1835), relate cet épisode, qui commença dans un grand enthousiasme - mais une terrible épidémie de peste mit fin à ce projet, et du même coup au séjour de la plupart des saint-simoniens en Égypte.

À la différence de l'expédition de Bonaparte, celle des saint-simoniens fut pacifique. Néanmoins, la figure du grand homme, qui avait réussi à revenir auréolé de son séjour oriental, alors même que son entreprise militaire avait été un échec, hantait les compagnons d'Enfantin. Barrault en témoigne à plusieurs reprises, dans son essai déjà cité, où il fait de Bonaparte une sorte de précurseur de leur propre volonté de concorde universelle:

Comme plus tard il prépara la fusion des populations européennes, alors Napoléon prépara la communion de l'Orient et de l'Occident. C'est pourquoi ici [en Égypte] le peuple a conservé avec une admiration sans rancune le souvenir de ses éclatantes victoires [...].

C'est lui qui au bout de ses baïonnettes apporta ici, en présent, la science européenne dont aujourd'hui les lumières se propagent; c'est lui qui songea à remuer, à rajeunir cette vieille

5 Sur cette entreprise, voir Régnier (1989). 
terre par un large mouvement de travail ; c'est encore lui qui rappela à l'Europe qu'elle avait par Soueys une route plus abrégée vers l'Inde. [...]

Ailleurs il a fait peut-être beaucoup d'ingrats; ici il reçoit un culte de reconnaissance. Si l'Égypte avait des saints, Napoléon en serait un; et peut-être, pour ces populations enthousiastes, est-il davantage (Barrault 1835, 449-450).

On reviendra tout à l'heure sur la phraséologie de l'union de l'Orient et de l'Occident, que bien des voyageurs reprendront en 1869. Insistons ici sur l'importance, pour les saint-simoniens, du souvenir de Napoléon, construit par Barrault comme le fondateur moderne de l'idée de Suez, c'est-à-dire comme un pont entre deux grandes parties du monde qui, ainsi que le disait Lamartine au même moment dans son Voyage en Orient, étaient entraînées dans un "inévitable rapprochement » (Lamartine 2000 [1835], 457).

Il est amusant de constater qu'on retrouve, dans un chapitre de l'ouvrage de Barrault consacré à la construction avortée d'un barrage sur le Nil, le récit d'une fête, celle, précisément, de Napoléon (le 15 août 1834), où des Égyptiens et des Français, incarnant " une même famille », constituent un " présage sublime de la communion de l'Orient et de l'Occident » (Barrault 1835, 455). Parmi les participants à cette fête figure Soliman Pacha, l'ex-colonel Sève, ancien officier de Bonaparte resté en Égypte après le départ de celui-ci, et qui, converti à l'islam, était devenu le chef de l'armée du vice-roi. Il porte d'ailleurs une série de toasts, dont le premier « À Napoléon, [...], non à l'empereur, mais au grand homme! » (Barrault 1835, 456), allusion à l'expédition d'Égypte à laquelle Barrault fera lui-même écho, un peu plus loin, dans son récit, de la façon suivante:

Si l'on n'avait pas autant abusé des Pyramides et des quarante siècles, peut-être aurait-il pu nous sembler que du haut de leur cime, car elles étaient sous nos yeux, Napoléon contemplait, non sans joie, cette fête improvisée, témoignage du développement de ses grandes pensées (Barrault 1835, 460).

Autrement dit, les saint-simoniens se conçoivent bel et bien, à l'instar du viceroi Mohammad Ali, comme les « exécuteurs testamentaires » de Bonaparte ${ }^{6}$. Construisant un barrage sur le Nil, ersatz du creusement de l'isthme de Suez, ils ne veulent pas seulement contribuer à la modernisation de l'Égypte, ils fantasment leur entreprise comme un acte fondateur de l'union entre Orient et Occident, dans une logique de réconciliation qui veut rompre avec la pensée d'une conflictualité ancestrale longtemps prévalente. On notera cependant que l'enjeu de toute cette entreprise est bel et bien, dans les termes mêmes de Barrault, la " régénération »

6 Soliman Pacha porte lui-même un toast «À Méhémet Ali, l'exécuteur testamentaire de Napoléon en Égypte! » (Barrault 1835, 456). 
(Barrault 1835, 455) de l'Égypte: c'est d'abord elle qu'il faut sauver, et non la France, qui apparaît, ici, dans son rôle « civilisateur » bien connu.

Notons enfin qu'on trouve aussi, parmi les participants à cette fête de Napoléon sur le Nil, Ferdinand de Lesseps en personne, alors « consul de France au Caire » (Barrault 1835, 452), et manifestement proche, à l'époque, des saint-simoniens, dont leur chef, le polytechnicien Prosper Enfantin, contribuera lui-même, sous le Second Empire, à rectifier l'erreur de l'ingénieur Le Père en démontrant l'égalité de niveau entre la mer Rouge et la Méditerranée, redonnant ainsi toute sa crédibilité scientifique au projet de canal. De Bonaparte à Lesseps, en passant par le séjour des saint-simoniens en Égypte, dans les années 1830, germe l'idée du creusement de l'isthme de Suez comme une sorte d'utopie réconciliatrice, dont on va examiner maintenant les éléments de discours à travers le témoignage d'un certain nombre de voyageurs invités pour les fêtes de 1869.

\section{Une utopie réconciliatrice}

Celui qui a le plus développé le thème de l'union des deux mers est sans doute l'historien de l'art Charles Blanc, dans un récit de voyage publié quelques années après l'ouverture du canal. Ce texte, intitulé Voyage de la Haute Égypte (1876), raconte tout à la fois la remontée du Nil qu'accomplirent un certain nombre d'invités, jusqu’à Louxor, Karnak et la Vallée des Rois, ce qui leur permit de visiter les grands sites pharaoniques, avec un guide rédigé pour l'occasion par l'égyptologue Mariette, et un chapitre final consacré spécifiquement aux fêtes d'ouverture du canal de Suez, autour de mi-novembre 1869. Le transport des voyageurs sur les villes du canal, depuis Le Caire, semble avoir posé des problèmes logistiques aux autorités égyptiennes. On peut d'ailleurs le comprendre, étant donné le nombre considérable d'invités. Une partie d'entre eux dut aller de la capitale à Alexandrie, en train, et de là prendre un bateau qui, en 18 heures, les amena à Port-Saïd en longeant la Méditerranée. Charles Blanc, de son côté, eut la chance d'échapper à ce trajet considérable en prenant, notamment avec un groupe d'artistes (Fromentin, Berchère, Tournemine), le train d'Alexandrie à Ismaïlia, car le chemin de fer entre Le Caire et les villes du canal de Suez n'était pas encore achevé. Voici en quels termes il parle de l'événement:

Il fallait vivre dans notre temps pour qu'un homme, après avoir conçu le gigantesque projet de percer l'isthme de Suez, vît ce projet se réaliser pleinement, pût assister au mariage de deux mers séparées durant tant de siècles, et naviguer lui-même sur des lacs étonnés de se voir traverser par des navires partis des ports de l'Europe pour aller aux Indes (Blanc 1876, 329; je souligne). 
Dans ce « mariage » maritime symbolique, qui est aussi celui de l'Orient et de l'Occident, l'Égypte, fût-elle incarnée par Ismaïl, occupe implicitement la position de la femme, puisque le projet de Suez est désigné comme celui d'un " homme », en l'occurrence Lesseps. On peut observer tout de suite une certaine asymétrie, à l'intérieur de ce couple: non seulement le khédive, qui est pourtant le partenaire égyptien sans lequel rien n’aurait pu être mené à terme, est oublié, mais, dans la formulation même des conséquences de l'existence du canal sur le commerce mondial, une seule direction est prise en compte, celle des navires « partis de l'Europe pour aller aux Indes ». Au fond, de la même façon que le mariage comme institution, sous le Second Empire, fait de la femme une mineure en droit, ce « mariage de deux mers », sans être un viol, traduit néanmoins un fantasme viril qui place l’Europe, représentée ici par celui qu'on surnommait « le Grand Français », en position dominante, voire conquérante.

Cette asymétrie sera quelque peu atténuée lorsque Blanc rapportera de manière enthousiaste l'acmé de la cérémonie d'ouverture, le 17 novembre 1869, à savoir le moment où des navires apparaîtront des deux côtés du canal pour se rencontrer dans le port d'Ismaïlia, marquant ainsi l'accomplissement de « l'union des deux mers » (Blanc 1876, 350). Mais là encore, les considérations qui suivent font de cette apparente communion un éloge qui renvoie en réalité au concepteur français du canal :

Je ne sais ce qui s'est passé en ce moment dans le cœur de M. de Lesseps. Mais nous étions tous très-émus et, pour mon compte, j'éprouvais un sentiment de fierté à voir comment le génie de l'homme avait su remanier notre planète, retoucher l'œuvre de la création, donner des ordres à l'Océan et s'en faire obéir (Blanc 1876, 351).

On voit que se met en place, à Suez, une mythologie moderne où Ferdinand de Lesseps apparaît comme un substitut du Dieu créateur. Le " génie de l'homme », ici, est moins une réalité anthropologique, à vocation universaliste, que le génie d'un homme, qui n'hésitera pas à s'auto-célébrer, dans des termes qui laissent songeur quant à la puissance de son narcissisme. Voyons comment il parle luimême de l'arrivée de l'Aigle, le yacht d'Eugénie sur lequel il se trouve, et qui est le premier navire à entrer dans le canal de Suez, le 17 novembre 1869:

[...] les acclamations éclatent, les vivats à l'impératrice se mêlent au nom salué de M. Ferdinand de Lesseps; l'Impératrice, elle-même, stimule cet élan; elle signale en quelque sorte aux spectateurs M. de Lesseps comme le premier sur qui doit se porter leur enthousiasme, et c'est dans un mouvement indescriptible de ravissement mêlé d'attendrissement que toute cette foule émue regarde passer ce beau navire, portant la bonne nouvelle de l'union accomplie de l'Occident et de l’Orient (Lesseps 1881, 339; je souligne). 
Parler de soi à la troisième personne, comme le fait ici Lesseps, est une fausse mise à distance. C'est une manière rhétoriquement habile de se faire doublement adouber, d'abord par les spectateurs, mais aussi par l'impératrice elle-même. Du coup, on comprend entre les lignes qu'Eugénie et Lesseps forment un nouveau « couple », le temps de la fête, autrement dit que Lesseps remplace symboliquement Napoléon III, resté en France. Cette cérémonie est une intronisation qui ne dit pas son nom. Ce qui n'empêche pas Lesseps de parler de «l'union [...] de l'Orient et de l'Occident » (Lesseps 1881, 339), reprenant ainsi la phraséologie saint-simonienne dont on a déjà vu des exemples chez Barrault.

Il s'agit là d'une véritable isotopie, qu'on retrouve, avec des variantes, chez nombre de voyageurs à Suez en 1869. Ainsi Charles Taglioni, attaché à la légation de Prusse à Paris, invité par le khédive Ismaïl, parle-t-il du canal comme $d u$ « trait d'union entre l'Occident et l'Orient » (Taglioni 1870, 236). Dans son récit de voyage, il cite intégralement le discours de Mgr. Bauër, représentant de l'Église catholique lors des fêtes d'inauguration de Suez, et par ailleurs confesseur de l'impératrice Eugénie. Dans ce discours, souvent cité et largement reproduit dans la presse contemporaine, le prélat parle de « ce seuil, qui fait de l'Orient et de l'Occident un seul et même monde » (Taglioni 1870, 217); il en appelle à leur « mutuelle fraternité » et incite ces deux espaces personnifiés, qui renvoient d'abord, pour lui, à l'islam et au christianisme, à « se rapprocher et s'étreindre ${ }^{7}$ » (Taglioni 1870, 218).

À ce thème de l'union entre l'Orient et l'Occident est associé celui du cosmopolitisme, entendu en général, dans ce contexte, comme la présence d'une assemblée de populations d'origines ethniques, culturelles et religieuses différentes. On touche ici à une difficulté conceptuelle qui se traduit souvent, dans les discours de l'époque, par une ambiguïté axiologique. En effet, comment peut-on à la fois reconnaître la diversité d'une « assistance auguste et cosmopolite » (Taglioni 1870, 217), comme l'écrit Mgr Bauër, et plaider en même temps pour un canal qui ouvrirait la voie à la « véritable civilisation » (Taglioni 1870, 221), dont on comprend rapidement qu'elle vient de la France? Le cosmopolitisme dont il est ici question est en réalité le rêve d'un Orient transformé par l'Occident. À preuve cette évocation du khédive, qualifié de "régénérateur » (Taglioni 1870, 222), et qui est remercié pour avoir accepté la présence d'églises et d'autels chrétiens à Suez, à côté de la tribune où se trouvent les représentants musulmans, mais en des termes révélateurs d'un profond eurocentrisme:

Pour la première fois depuis douze siècles, la foi chrétienne peut élever, en face du croissant, à ciel ouvert, sa voix pour prier et pour bénir. [...]; merci, d’un cœur ému, au nom du

7 Sur ce discours, voir Lançon (2019, 121-135). 
christianisme; merci au nom de la France, au nom de l'Europe; merci au nom de toute l'humanité, dont les destinées font un grand pas en ce moment, grâce à Votre Altesse, qui veut le bien, et à Dieu qui le bénit (Taglioni 1870, 230).

Et, pour faire bonne mesure, Mgr Bauër compare Ferdinand de Lesseps à Christophe Colomb ${ }^{8}$, ce qui en dit long sur la dimension impérialiste et prosélyte sousjacente à l'entreprise de Suez, au moins pour certains invités, et sur le rôle que l'Église catholique joua comme appui du pouvoir politique et économique, en l'occurrence celui du Second Empire.

On retrouve le même type de double discours chez un voyageur contemporain invité aux fêtes de Suez, Émile de la Bédollière, écrivain, traducteur, journaliste au National à partir de 1869. « Ce qui a fait le charme de ce jour de féerie », écrit-il à propos du 17 novembre, «ç'a été l'entrain, l'animation, la cordialité fraternelle qui régnaient entre tant d'hommes de races diverses » (Bédollière 1870, 31-32); mais dès lors qu'il visite la capitale égyptienne, quelques jours plus tard, il dénonce pêle-mêle les " horribles clameurs » accompagnant les cortèges de noces ou d'enterrements et les « misérables fanatiques » fêtant la rupture du jeûne musulman, ce qui le conduit à fuir « le brouhaha du Caire » (Bédollière 1870, 63-64).

Même sentiment d'un grand tumulte désagréable, cette fois-ci associé à la présence des cheikhs arabes lors des fêtes de Suez, chez Florian Pharaon, ancien interprète de l'armée française en Algérie et correspondant du journal La France, favorable à Napoléon III :

Les campements arabes sont épars sur les bords du canal et sur les rives du lac. Les chevaux, entravés, hennissant au devant des tentes, et les dromadaires bruyants, accroupis, frappent l'air de leurs cris plaintifs. Les chefs des tentes, étendus sur des tapis aux couleurs vives, écoutent, calmes et silencieux, la musique tapageuse des orchestres ambulants du désert : - un ensemble parfaitement discordant de clarinettes criardes dominant le vacarme des tambourins. Le bruit règne en maître dans cette cité de toile; l'oreille, assourdie par cette débauche de sons de toute nature, finit enfin par s'accoutumer à ce tumulte. Il y a ici tous les échantillons de la race sémitique; tous les peuples soumis à l'islamisme ont envoyé des représentants: le Persan frôle l'homme du Maroc et celui de Zanzibar; l'habitant de l'Arabie a dressé sa tente bariolée à côté du cône rayé de l'Indien. Ajoutez à cette agglomération les campements préparés par le Khédive pour recevoir les invités de l'Europe et de l'Amérique, et vous aurez une idée exacte de ce qu'a pu être la tour de Babel (Pharaon 1872, 45-46).

8 « Proclamons enfin que jusqu'à l'extrême déclin des âges, de même que le nouveau monde découvert au quinzième siècle dira à jamais, à l'oreille de toute postérité, le nom de l'homme de génie qui s'appela Christophe Colomb, de même ce canal des deux mondes redira à jamais le nom d'un homme qui vécut au dix-neuvième siècle, ce nom que je suis heureux de jeter sur cette plage aux quatre vents du ciel, le nom de Ferdinand de Lesseps » (Taglioni 1870, 225). 
La conclusion de cette énumération dit bien ce que signifie, pour Florian Pharaon, cette juxtaposition de peuples divers : une multiplication de langues et de « races » produisant un sentiment de disharmonie - l'entrée dans un monde babélien. (Notons au passage que la musique arabe, associée aux cris des animaux, participe elle-même, intrinsèquement en quelque sorte, de cette cacophonie suezienne, illustrant ainsi doublement le «discours orientaliste » dénoncé par Said ${ }^{9}$ ).

Cependant, la mosaïque ethnique des fêtes de Suez n’a pas toujours produit un effet désagréable sur les voyageurs français. Charles Blanc, par exemple, se livre à un morceau de bravoure descriptive qui fait de ce monde en réduction une expérience positive du divers ${ }^{10}$ - mais il transforme finalement l'énumération des invités orientaux en un tableau destiné à séduire le lecteur par son exotisme orientalisant ( « Il en était venu de Tanger, de Méquinez, d’Alger, de Bengazi, de Senaar, de toutes les contrées où l'on fabrique des bijoux, des chibouques, des armes, où l'on dit des prières sur les tapis ») (Blanc 1876, 341). Cela dit, dès lors qu'il entre en contact avec certains des invités orientaux, Charles Blanc marque assez vite une distance critique. Ainsi, lorsqu'un cheikh lui offre le spectacle de derviches tourneurs, il décrit ceux-ci comme des moines qui « se trémoussaient de gauche à droite et de droite à gauche, de manière à se procurer, par ces mouvements rhythmés, une sorte d'extase que favorise une musique religieuse et monotone » (Blanc 1876, 348; je conserve l'orthographe de l'époque) : le ton voltairien renvoie à une tradition anticléricale bien vivante en France, mais il sert surtout, ici, à discréditer une expérience religieuse orientale, en l'occurrence un rituel appartenant à la mystique soufie, ce qu'ignorent d'ailleurs nombre de voyageurs français, qu'ils soient ou non sensibles aux rituels de cette forme d'islam hétérodoxe (voir Moussa 2014).

L'expérience cosmopolitique que procurent les fêtes du canal de Suez, expérience entendue comme la mise en contact massive, dans un espace-temps bien déterminé, de populations orientales et occidentales d'habitude séparées les unes des autres, reste majoritairement perçue par les voyageurs français selon des grilles de lecture fortement eurocentriques.

\footnotetext{
9 E. Said écrivait d'ailleurs: « Dans l'idée du canal de Suez, nous voyons la conclusion logique de la pensée orientaliste » $(1980,110)$.

10 J'ai commenté cela dans Moussa (2019b, 75-93).
} 


\section{Les absents de l'Histoire}

L'eurocentrisme des voyageurs français se manifeste aussi dans la façon dont ils passent sous silence, ou sous-évaluent, un certain nombre d'acteurs des fêtes de 1869. Les grands absents de ces cérémonies sont les fellahs, les paysans égyptiens qui payèrent de leur vie, par milliers, le creusement du canal, pendant les premières années du chantier, sous Saïd Pacha, entre 1859 et 1863, chantier qui fut mené, à cette époque, entièrement avec une main d'œuvre égyptienne, à peine rémunérée, sous équipée, sujette aux maladies, et souvent amenée de force, à la suite de marches épuisantes dans le désert, dans le cadre de la corvée à laquelle elle était soumise - avant que les fellahs ne soient remplacés à la fois par des ouvriers étrangers mieux payés et secondés par des machines, dont les fameuses dragues amenées d'Europe ${ }^{11}$.

Certes, Charles Taglioni, dans Deux mois en Égypte (1870), rappelle que « vingt mille fellahs étaient remplacés mensuellement par un nombre égal de nouveaux travailleurs » pendant les premières années du chantier de Suez (Taglioni 1870, 248). Mais rien n'est dit sur leurs conditions de vie, et c'est finalement la littérature contemporaine de fiction qui, timidement, parle du rôle des paysans égyptiens dans le creusement de l'isthme, sans d'ailleurs mettre en cause le bien-fondé de l'entreprise - on pense notamment au Fellah (1869) d'Edmond About, où il apparaît que le père du héros est mort pendant le creusement de l'isthme, mais que son fils en attribue finalement la responsabilité uniquement au système égyptien de la corvée, et qu'il croit aux bienfaits du canal pour son pays (voir Moussa 2019a, 55-72). Quant à Mgr Bauër, dont Taglioni reproduit le discours tenu à Suez, il mentionne certes les fellahs, mais c'est pour les récupérer immédiatement comme des " pionniers conscients ou inconscients de la Providence », qui ont eu « la gloire de donner un coup de pioche pour ouvrir la route magnifique où passeront désormais à jamais la paix et la justice, la lumière et la vérité, c'est-à-dire, au sens le plus élevé, la véritable civilisation » (Taglioni 1870, 221).

Il est frappant de constater que les paysans égyptiens sont véritablement les absents de la fête. Lorsqu'ils sont aperçus en tant qu'êtres réels, c'est-à-dire dans leurs villages, ou travaillant aux champs, c'est justement dans un autre contexte, c'est-à-dire hors des villes du canal. Émile de la Bédollière en voit lorsqu'il prend le train de retour, du Caire à Alexandrie, dans le Delta. Les quelques mots méprisants qu'il leur accorde témoignent à la fois de son ignorance et, sans doute, d'un mépris de classe qui s’ajoute au préjugé « orientaliste »: « Il [le fellah] mène une

11 Sur cette question, voir Farouk (2019, 137-149). 
existence purement animale, pratique machinalement sa religion, n'ambitionne rien, ne pense à rien » (Bédollière 1870, 78).

Les Arabes, c'est-à-dire essentiellement les cheikhs des tribus nomades invités à participer aux fêtes de Suez par des spectacles équestres (Taglioni 1870, 263), sont un peu plus présents, mais, au-delà du pittoresque de circonstance, le préjugé ethnique et culturel, ainsi que l'attitude méprisante qui va de pair, règnent en maître. Lesseps, par exemple, cherche à montrer l'adhésion des populations indigènes à son projet, mais il en profite pour stigmatiser « les sons plus bruyants qu'harmonieux de tous les instruments de musique que les Arabes possèdent pour témoigner leur allégresse » (Lesseps 1881, 339). Charles Blanc, qui se montre pourtant sensible aux charmes de la diversité culturelle, s'effarouche de celleci dès lors qu'il y est directement confronté, comme lorsqu'il est invité à « un dîner arabe, où il [lui] fallut, faute de fourchettes et de couteaux, dépecer les viandes crues avec [ses] doigts » (Blanc 1876, 348), ainsi qu'il l'explique, sûr de faire sourire son lecteur français par la confrontation de deux modèles de savoirvivre dont il n'est pas nécessaire de préciser lequel est supérieur à l'autre. Florian Pharaon, de son côté, s'en donne à cœur joie dans la mise en scène humoristique des chocs culturels auxquels peuvent donner lieu la confrontation de deux mondes différents :

Le Khédive avait fait venir à ce bal [dans son palais d'Ismaillia] tous les Cheik-el-Belad, honorables fonctionnaires que je ne saurais mieux assimiler qu'à nos braves maires de villages. Quatre d'entre eux, appartenant aux tribus nomades, dans leur promenade admirative à travers les salons, se fourvoient dans le sanctum sanctorum destiné aux femmes.

La vue du boudoir les enchante, et ils se parfument abondamment avec les essences déposées sur les consoles; l'un d'eux prend en main une boîte de poudre de riz, en manie la houppe, s'informe auprès de la femme de chambre et se saupoudre imperturbablement la face; ses trois compagnons l'imitent; puis tous quatre font leur entrée dans les salons. L'on devine l'effet produit ${ }^{12}$ (Pharaon 1872, 47-48).

On a ici une illustration parfaite de ce que Homi Bhabha appelle mimicry, ce processus d'imitation dévaluée que le discours impérialiste impute à l' « Autre » (l’Oriental, le colonisé, le subalterne...) (Bhabha 1994). C'est là, au fond, une situation de double bind dont ces Bédouins de Suez se trouvent prisonniers. Car pour devenir « civilisés », il faut bien qu'ils tentent d'imiter les mœurs euro-

12 Voir également la façon dont Roberto Morra di Lavriano, militaire, homme politique puis diplomate italien, rend compte de la présence d'invités arabes lors de cette « fête cosmopolite très pittoresque » que représente le bal offert par le khédive à Ismaïlia : « Ils [les cheikhs] paraissaient fort incommodés d'avoir dû mettre des chaussettes pour la circonstance: dans tous les coins, côtoyant les élégantes, on pouvait les voir, avec ce calme qu'ils ont, comme inconscients de ce qui les entourait, se palper les pieds comme à l’ordinaire » (Morra di Lavriano 1997, 123). 
péennes. Mais, précisément parce qu'ils sont des « sauvages » (même fascinés par l'Europe), cette imitation restera à jamais imparfaite. Le mythe bédouin, reposant sur un imaginaire de la liberté que nombre de voyageurs français ont projeté sur les Arabes nomades en allant parfois jusqu'à s’identifier à eux, en particulier à l'époque romantique (voir Moussa 2016), se dégrade peu à peu, dans la seconde moitié du XIX ${ }^{\mathrm{e}}$ siècle, au point d'inverser son sens et d'en revenir, fût-ce sous une forme humoristique, comme ici, à la critique traditionnelle de la " sauvagerie ${ }^{13}$ ».

La dernière catégorie d' " oubliés », et non des moindres, est celle des invités orientaux. Curieusement, on sait très peu de choses sur eux, alors qu'ils étaient au moins des centaines, voire des milliers selon certains témoignages. Ainsi la présence du sultan de Zanzibar est-elle annoncée par une lettre qu'il adresse à Lesseps et qui est reproduite dans un numéro de L'Isthme de Suez, le journal créé pour promouvoir les travaux du chantier et les intérêts de la Compagnie universelle du canal maritime de Suez ${ }^{14}$. A-t-il laissé des traces de son séjour? Seule une enquête approfondie, avec des spécialistes de différentes langues orientales, pourrait répondre à ce type de question portant sur la présence africaine, mais aussi ottomane, indienne, etc., lors des fêtes de 1869. On en est donc réduit, pour le moment, à interroger les documents issus d'invités européens à Suez. Or ceuxci restent très lacunaires et signalent au mieux, comme le fait le Voyage de Charles Blanc, que « le vice-roi [sic, pour le khédive] avait convié à ses fêtes tous les grands personnages de l'Islam » (Blanc 1876, 341). Qui sont-ils? Blanc ne le dit pas, et ne cherche visiblement pas à le savoir. Cette présence des invités orientaux est du reste assez vite réduite à une foule indifférenciée, si ce n'est par sa couleur de peau (« c'était une macédoine de populations jaunes, noires ou cuivrées... »; Blanc 1876, 349), du moins à une suite de noms de lieux exotiques - Tanger, Méquinez, Alger, Bengazi, Senaar ${ }^{15}$.

Le seul personnage de marque qui soit nommé par certains voyageurs est Abd el-Kader, le célèbre émir algérien qui avait organisé la résistance à la colonisation française de l'Algérie, avant de se rendre, en 1847, puis d'être emprisonné plusieurs années en France, et d'être envoyé en exil à Damas, où il joua un rôle favorable à la France, notamment pendant les massacres anti-chrétiens, en 1860.

13 Voir également É. de la Bédollière, dont la représentation des Arabes nomades qu'il aperçoit à Sakkara, à l'occasion d'une excursion à l'issue des fêtes de Suez, est aussi négative que celle des fellahs: « La horde de Bédouins qui s’est établie là pour rançonner les voyageurs et s'est ruée sur nous comme une proie, se retire les mains pleines de batschisch [= bakchich, le pourboire] » $(1870,74)$.

14 Voir la lettre du sultan de Zanzibar traduite en français, datée du 29 juin 1869, et reproduite dans le numéro 316 de L'Isthme de Suez.

15 Voir ci-dessus et Blanc (1876, 341). 
Abd El-Kader fut donc spécialement honoré en figurant, seul invité « Oriental », dans la tribune d'honneur à Port-Saïd ${ }^{16}$. Le tout jeune égyptologue genevois Édouard Naville, qui joua le rôle d'assistant auprès de l'égyptologue prussien Lepsius, grâce à qui il fut lui aussi invité aux fêtes de Suez, rend compte de cette présence particulière dans une lettre adressée à ses proches:

Mais l'homme qui m'a le plus intéressé à voir, c'est Abd el-Kader, qui est encore jeune et remarquablement beau. Il se tenait du reste assez à part, étant l'hôte du sultan, et non pas du vice-roi (Berchem 1989, 110).

Charles Blanc, lui aussi, est visiblement fasciné par ce personnage, qui fait une apparition aussi théâtrale que fugitive, au milieu des invités du bal offert par le khédive dans son palais d'Ismaïlia: "Abd el-Kader parut. Ses grands yeux noirs, étincelants, étaient voilé de douceur. Un souper fut servi à quinze cents personnes » (Blanc 1876, 351).

Le ralliement à la France de l'ancien résistant algérien ne s'oublie pas. Abd el-Kader est donc l'exception qui confirme la règle. Il est honoré non seulement par la place qui lui est attribuée, proche des souverains européens, mais aussi par le fait qu'il soit le seul invité arabe de marque dont le nom figure dans les récits de voyageurs français. Par comparaison, on a de nombreuses mentions des personnalités occidentales dont le navire suit celui de la princesse Eugénie - l'empereur austro-hongrois François-Joseph, le prince royal de Prusse Frédéric-Guillaume, les princes des Pays-Bas et de Hanovre, sans parler des ambassadeurs de la plupart des pays européens invités au banquet d'Ismaïlia offert par le khédive, ou encore des différentes listes d'invités (que ce soit ceux d'Ismaill, ceux de Lesseps, ou encore ceux du conseil d'administration de la Compagnie de Suez), listes de noms, notons-le au passage, exclusivement français, et que Jean-Marie Carré a reconstituées, en se basant à la fois sur le témoignage de voyageurs contemporains et sur des journaux de l'époque (Carré 1990 II [1956], 359-367, Appendice I).

Nommer, c'est faire exister. Mais cela ne suffit pas toujours pour poser les bases d'une mémoire historique partagée. En ce sens, le « cosmopolitisme » des fêtes de Suez, une expression qu'on trouve sous la plume de nombre de voyageurs français, est profondément incomplet, car il se limite le plus souvent à une vision superficielle - au mieux exotisante, au pire raciste, le plus souvent " orientaliste » au sens saïdien du terme -, et qui fait en tout cas l'impasse sur la part proprement orientale de l'événement "Suez », aux dépens de ce qui était célébré, de manière trompeuse, comme le triomphe universel du progrès, de la paix et du commerce unissant les peuples.

16 Sur les liens d'Abd el-Kader avec Lesseps, qui avait envisagé de lui offrir un domaine aux bords du canal de Suez, voir Faruk Bilici (2019, 155-165). 


\section{Une voix discordante}

Pour terminer, on évoquera brièvement la figure de l'écrivain Théophile Gautier, qui fut invité par le khédive Ismaïl aux fêtes d'ouverture de Suez, mais qui ne put accomplir le voyage de la Haute-Égypte, s'étant luxé une épaule dans le bateau qu'il avait pris pour traverser la Méditerranée. Il publia à son retour un début de récit de voyage de ce séjour, sous la forme de six feuilletons parus dans le Journal Officiel en 1870 (voir Gautier 2016). Curieusement, malgré un court texte resté manuscrit et intitulé « L'isthme de Suez », Gautier ne dit rien des cérémonies proprement dites, auxquelles il assista pourtant, on le sait par sa correspondance. Rien, donc, de l'émotion (certains pleurent, comme le vice-amiral Pâris ${ }^{17}$ ) qui étreint l'ensemble des spectateurs lorsqu'ils voient arriver à Ismaïlia le yacht de l'impératrice, laquelle, d'après Fromentin, étant elle-même particulièrement émue, en oublia de serrer la main de Lesseps ${ }^{18}$. Tout aussi étrange: sur l'ensemble de ce récit de voyage, fût-il court et inachevé, Gautier ne prononce pas une seule fois le nom de Ferdinand de Lesseps, dont on a vu qu'il est considéré par certains invités comme une sorte de Dieu vivant du progrès industriel et technologique. Il est toujours délicat d'interpréter des silences. Mais celui-ci est particulièrement assourdissant. Mon hypothèse est que Gautier ne croyait pas au discours idéalisant de l' " union des deux mers », ou plus exactement qu'il avait pressenti, comme Edward Said le dira explicitement et avec force une centaine d'années après lui, que «Ferdinand de Lesseps avait fait fondre l'identité géographique de l'Orient en entraînant (presque littéralement) l'Orient dans l'Occident » (Said 1980, 111). De fait, on sait que le canal de Suez fut une catastrophe financière pour l'État égyptien, qui s'endetta, finit par vendre ses parts de la Société dite universelle à l'Angleterre, laquelle n'eut pas de peine à occuper militairement l'Égypte, à partir de 1882 (voir Piquet 2009). Le « cosmopolitisme » que mettent en avant nombre de voyageurs français, en 1869, est en réalité fortement eurocentré: il annonce un monde où l'Orient lui-même s'engage dans un processsus de modernisation, donc d'européanisation, une évolution dont Gautier, amoureux de la différence, est le témoin affligé.

17 Le fait est rapporté par Ferdinand de Lesseps (1881, 333-334).

18 «L'impératrice sur sa haute dunette agite son mouchoir. Elle a près d'elle M. de Lesseps, elle oublie de lui serrer la main devant ce grand public, venu de tous les points de l'Europe, et dont l'émotion est extrême » (Fromentin 1984, 1091). 


\section{Références bibliographiques}

Barrault, Émile. Occident et Orient. Études politiques, morales et religieuses pendant 18331834 de l'ère chrétienne, 1249-1250 de l'Hégyre. Paris: Desessart et Pougin, 1835.

Bédollière, Émile de la. De Paris à Suez. Souvenirs d'un voyage en Égypte. Paris: Librairie Georges Barba, 1870.

Berchem, Denis van. L'Égyptologue genevois Édouard Naville. Genève: Georg et Journal de Genève, 1989.

Bhabha, Homi K. The Location of Culture. London: Routledge, 1994.

Bilici, Faruk. Le Canal de Suez et l'empire ottoman. Paris: CNRS Éditions, 2019.

Blanc, Charles. Voyage de la Haute Égypte. Observations sur les art égyptien et arabe. Paris: Renouard, 1876.

Carré, Jean-Marie. Voyageurs et écrivains français en Égypte, Vol. 2. Le Caire: IFAO, 1990 [1956] [1932-1933].

Descotes, Maurice. La Légende de Napoléon et les écrivains français du XIXe siècle. Paris: Lettres modernes, 1967.

Farouk, May. «La corvée lors du creusement de l'isthme. Guerre des discours et représentation romanesque». Les imaginaires du canal de Suez. Éd. Sarga Moussa et Randa Sabry. Sociétés et Représentations 48 (2019): 137-149.

Fromentin, Eugène. «Carnets du voyage en Égypte». «Carnet I ». CEuvres complètes. Éd. Guy Sagnes. Paris: Gallimard (« Bibliothèque de la Pléiade »), 1984 [1869], 1049-1111.

Gauthier, Gilles et Claude Mollard. Éds. L'Épopée du canal de Suez. Paris: Institut du Monde Arabe/Musée d'Histoire de Marseille/Gallimard, 2018.

Gautier, Théophile. Voyage en Égypte. Éd. Sarga Moussa, Vol. IV, 6 de Euvres complètes. Paris: Champion, 2016 [1870].

L'Isthme de Suez. Journal de l'union des deux mers, 316. Lettre du sultan de Zanzibar, 1869.

Lamartine, Alphonse de. Voyage en Orient, Éd. Sarga Moussa. Paris: Champion, 2000 [1835].

Lançon, Daniel. «Tentatives de réinvention d'une géographie sacrée de l'isthme lors de la création du canal de Suez (1855-1870)». Les imaginaires du canal de Suez. Éd. Sarga Moussa et Randa Sabry. Sociétés et Représentations 48 (2019): 127-141.

Le Père, Jean-Marie. «Mémoire sur la communication de la mer des Indes à la Méditerranée par la mer Rouge et l'isthme de Soueys». Description de l'Égypte. Vol. XI: État moderne. Éd. Edme-François Jomard. Paris: Panckoucke, 1822 [1809], 37-369.

Lesseps, Ferdinand de. Lettres, journal et documents pour servir à l'histoire du canal de Suez, Vol. V. Paris: Didier, 1881.

Morra di Lavriano, Roberto. Journal de voyage en Égypte. Inauguration du canal de Suez. Éd. Alberto Silotti et Alain Vidal-Naquet. Trad. Nicole Sels. Paris: Gründ, 1997.

Moussa, Sarga. «Voix d'islam, résonances viatiques. Perception de la prière musulmane chez quelques voyageurs au XIX ${ }^{\prime}$ ». Viatica 1 (2014). http://revues-msh.uca.fr/viatica/index. php?id=423 ( $1^{\text {er }}$ mai 2020$)$.

-. Le Mythe bédouin chez les voyageurs français auX XVIII et XIXe siècles. Paris: Presses de l'Université Paris Sorbonne, 2016.

-. «Enjeux et difficultés d'un mariage interculturel à Suez: Le Fellah (1869) d’Edmond About». Dialogues interculturels à l'époque coloniale et postcoloniale. Représentations littéraires et culturelles. Orient, Maghreb et Afrique occidentale (de 1830 à nos jours). Éds HansJürgen Lüsebrink et Sarga Moussa. Paris: Kimé, 2019a, 55-72. 
-. «Revisiter le mythe de Suez. Écrivains et voyageurs français en Égypte autour de 1869». Voix d'Orient. Mélanges offerts à Daniel Lançon. Éd. Ridha Boulaâbi. Paris: Geuthner, 2019b, 75-93.

- et Randa Sabry. Éds. Les imaginaires du canal de Suez. Représentations littéraires et culturelles, Vol. 48 de Sociétés et Représentations, 2019.

Pharaon, Florian. Le Caire et la Haute Égypte. Paris: Dentu, 1872.

Piquet, Caroline. Histoire du canal de Suez. Paris: Perrin, 2009.

Régnier, Philippe. Les Saint-Simoniens en Égypte, 1833-1851. Le Caire: Amin F. Abdelnour/ Banque de l'Union Européenne, 1989.

Said, Edward. L'Orientalisme. L'Orient créé par l'Occident. Trad Catherine Malamoud. Paris: Seuil, 1980.

Taglioni, Charles. Deux mois en Égypte. Journal d'un invité du khédive. Paris: Amyot, 1870. 
